\title{
Headache in cerebral venous thrombosis: incidence, pattern and location in 200 consecutive patients
}

\author{
Mohammad Wasay · Suleman Kojan • \\ Alper I. Dai · George Bobustuc · Zubair Sheikh
}

Received: 5 October 2009/ Accepted: 31 December 2009/Published online: 29 January 2010

(C) Springer-Verlag 2010

\begin{abstract}
We analyzed the incidence, pattern and location of headache in consecutive 200 patients with proven diagnosis of CVT to identify an association between localization of headache and site of sinus involvement. Headache was present in $136(68 \%)$ patients. The duration of headache (reported in 128 patients) was reported as acute (1-3 days), 81 patients (60\%); sub-acute (4-14 days), 33 patients (24\%); and chronic (more than 14 days), 14 patients (10\%). The quality of headache (reported in 72 patients) was reported as throbbing 12 (9\%), band like 27 (20\%), thunderclap 7 (5\%), and other (pounding, exploding, stabbing, etc.) 26 (20\%). The location of headache (reported in 101 patients) was reported as unilateral (one side of head) 48 (37\%), localized (frontal, temporal, occipital, and neck) 25 (19\%), and diffuse
\end{abstract}

These findings were presented in preliminary form at International Headache Congress at Kyoto, Japan in 2005.

\footnotetext{
M. Wasay $(\bowtie)$

Department of Medicine/Neurology,

The Aga Khan University,

Stadium Road, Karachi 74800, Pakistan

e-mail: mohammad.wasay@aku.edu;

mohammadwasay@hotmail.com

S. Kojan

King Abdulaziz Medical City,

Riyadh, Saudi Arabia

\author{
A. I. Dai \\ Gaziantep University, Gaziantep, Turkey \\ G. Bobustuc \\ University of Texas, Houston, TX, USA \\ Z. Sheikh \\ University of Michigan, Ann Arbor, MI, USA
}

(whole head) 28 (20\%). 43 (32\%) patients had normal neurological examination (normal mental status, cranial nerves, motor and sensory examination with down going planters). $93(68 \%)$ patients have abnormal findings on neurological examination including papilledema 29 (15\%) patients, altered mental status $38(19 \%)$, and focal neurological deficit $45(22 \%)$ patients. There was no association between headache and presence of hemorrhage on CT and MRI $(P=0.1)$ or hydrocephalus $(P=0.09)$. There was no association between localization of headache and site of sinus thrombosis except sigmoid sinus thrombosis, where 17 out of 28 patients with involvement of sigmoid sinus alone or in combination with transverse sinus had pain in the occipital and neck region $(P<0.05)$. There was no association between lateralization of pain and site of thrombosis $(P=0.66)$.

Keywords Headache - Pattern ·

Cerebral venous thrombosis

\section{Introduction}

Headache is the most common presenting symptom of cerebral venous thrombosis present in $70-90 \%$ patients [13]. It may be the sole complain or abnormality in a number of patients. One study from France reported 17 (15\%) out of 123 patients with confirmed CVT had headache with normal neurological examination and normal CT scan [4].

Few studies have looked at the characteristics and localizing value of headache in CVT. The headaches in CVT could be acute, sub-acute or chronic. The presentation of headache could mimic thunderclap headache [5], migraine [6], orthostatic headache [7], cluster headache [8], headache related to increased intracranial pressure, and 
diffuse tension type headache. Previous studies have identified a positive correlation between acute onset severe headache and CVT [9].

Previous studies did not evaluate a correlation between localization of headache and site of sinus involvement We hypothesized that patients with isolated transverse or sigmoid sinus thrombosis have more localized pain as compared to diffuse pain in patients with superior sagittal sinus or multiple sinus thrombosis. The objective of our study was to analyze the frequency, pattern and location of headache and its relationship to site of sinus involvement or presence of hemorrhage in a large cohort of patients.

\section{Methods}

Patients with diagnosis of CVT were identified by ICD-9 coding system at ten centers in the United States during a 10-year period by retrospective chart review (1991-1997) or prospective enrollment (1997-2001). A total of 311 charts were reviewed and 111 excluded from study (61 due to age less than 8 years, 50 due to unconfirmed diagnosis). The data of 200 patients analyzed in the study were obtained through retrospective review $(n=108)$ and prospective collection $(n=92)$. Risk factors, presentation, treatment and outcome of these patients have been previously published. The diagnosis of CVT was confirmed by cerebral angiogram (41 patients), magnetic resonance imaging and venogram (89 patients), MRI (44 patients), and CT scan (26 patients). The diagnosis of CVT on brain imaging including CT scan was based on established criteria [10]. The data were collected on a pre-specified questionnaire for all patients.

\section{Results}

Age range was 8-82 years (mean 37 years). 18 (9\%) were less than 12 years old. $120(60 \%)$ were women. Headache was the most common feature, present in 136 (68\%) patients. It was less common in pediatric patients, $7 / 18$ (36\%). $22 \%$ of these patients had previous history of headache including migraine, tension headache, and cluster headache. Duration of headache ranged from 1 day to 6 months (mean 3 days). The characteristics of headache are given in Table 1.

$43(32 \%)$ patients had normal neurological examination (normal mental status, cranial nerves, motor and sensory examination with down going planters). 93 (68\%) patients have abnormal findings on neurological examination including papilledema 29 (15\%) patients, altered mental status 38 (19\%), and focal neurological deficit $45(22 \%)$ patients. All patients $(n=200)$ underwent brain imaging
Table 1 Headache characteristics $(n=136$ patients)

\begin{tabular}{ll}
\hline Duration & \\
Acute (1-3 days) & 81 patients $(60 \%)$ \\
Sub-acute (4-14 days) & 33 patients $(24 \%)$ \\
Chronic (more than 14 days) & 14 patients $(10 \%)$ \\
Unclear & 8 patients $(6 \%)$ \\
Quality (reported in 72 patients) & \\
Throbbing & $12(9 \%)$ \\
Band like & $27(20 \%)$ \\
Thunderclap & $7(5 \%)$ \\
Other & $26(20 \%)$ \\
Location (reported in 101 patients) & \\
Unilateral & $48(37 \%)$ \\
Localized & $25(19 \%)$ \\
Diffuse & $28(20 \%)$ \\
\hline
\end{tabular}

including post-contrast MRI 34, non-contrast MRI 90, post-contrast CT scan 67, and non-contrast CT scan 9. 49 (25\%) patients had intracerebral hemorrhage. None of these patients had sub-arachnoid hemorrhage. Other neuroimaging findings included non-hemorrhagic infarcts 55 (27\%), hydrocephalus 6 (3\%), mass effect $27(13 \%)$, midline shift $12(6 \%)$, vasogenic or diffuse cerebral edema $18(9 \%)$, and contrast enhancement $13(6 \%) .19$ patients $(10 \%)$ in our series had Benign intracranial hypertension. Site of sinus involvement (location of thrombosis) is provided in Table 2

There was no association between headache and presence of hemorrhage on CT and MRI $(P=0.1)$ or hydrocephalus $(P=0.09)$. There was no association between localization of headache and site of sinus thrombosis except sigmoid sinus thrombosis, where 17 out of 28 patients with involvement of sigmoid sinus alone or in combination with transverse sinus had pain in the occipital and neck region $(P<0.05)$. There was no association

Table 2 Location of thrombosis

\begin{tabular}{ll}
\hline Superior sagittal sinus (SSS) alone & $59(29 \%)$ \\
Transverse sinus (TS) alone & $44(22 \%)$ \\
Straight sinus (SS) alone & 1 \\
Sigmoid sinus alone & $9(5 \%)$ \\
Jugular vein (JV) alone & 1 \\
Internal cerebral vein/vein of Galen & $3(2 \%)$ \\
Cortical vein & $7(4 \%)$ \\
SSST + TS & $35(17 \%)$ \\
TS + sigmoid sinus & $19(10 \%)$ \\
TS + SS + JV & $2(1 \%)$ \\
Bilateral TS & $15(7 \%)$ \\
Superficial + deep venous system & $5(3 \%)$ \\
\hline
\end{tabular}


between lateralization of pain and site of thrombosis $(P=0.66)$.

\section{Discussion}

There is no identifiable, uniform, recognizable pattern of headache in CVT, but more commonly the "headache profile" is as follows: new onset, sub-acute to rapidly progressive over a few days, intermittent initially to become a constant headache later, mostly unilateral or localized to any region of the head and usually throbbing in nature [11]. The mechanism for headache in CVT is not well understood. Multiple mechanisms including a combination of raised intracranial pressure, subarachnoid hemorrhage, stretching of nerves in sinus walls and inflammation of sinus walls are most likely responsible for variable patterns of headache among these patients. Most patients had diffuse headache without any significant association to the presence of hemorrhage and location of thrombosis. Only significant correlation was involvement of sigmoid sinus thrombosis and occipital pain. This is probably related to inflammation and stretching of sigmoid sinus walls due to thrombus. Overall, unilateral headache was twice more common than diffuse or localized headache but unilateral pain had no association with site of thrombosis. These findings suggest that multiple mechanisms are responsible for these headaches.

The study is limited by partly retrospective enrollment. Presence and duration of headache were well reported in charts but pattern and location of headache were only reported in one-third of patients. Duration of headache was recorded from patient's history and could be affected by memory bias. It is often impossible to state the day of onset of CVT, which adds to the methodological problem addressing CVT and headache correlation. A major limitation of the study is the lack of use of a validated headache questionnaire for current and previous headache types. The diagnosis of CVT could be challenging in patients with history of migraine, complains of headache, and normal neurological examination. In our series, $68 \%$ patients with headache had associated neurological abnormalities, but in $32 \%$ patients headache was the sole abnormality. A differential diagnosis of CVT and MRI should be considered in all new acute or sub-acute headaches. Special attention should be paid to transverse and sigmoid sinus if the patient complains of localized occipital and neck pain. A future prospective study with serial interviews of the CVT patients using a validated headache questionnaire and relating the responses to neuroimaging findings may be helpful in confirming these findings.

Acknowledgments Authors are thankful to Professor J. Stam, University of Amsterdam, Netherlands for manuscript review and suggestions.

Conflict of interest None.

\section{References}

1. Ferro JM, Canhao P, Stam J, Bousser MG, Barinagarrementeria F (2004) ISCVT Investigators. Prognosis of cerebral vein and dural sinus thrombosis: results of the International Study on cerebral vein and dural sinus thrombosis (ISCVT). Stroke 35(3):664-670

2. Wasay M, Bakshi R, Bobustuc G, Kojan S, Sheikh Z, Dai A, Cheema Z (2008) Cerebral venous thrombosis: analysis of a multicenter cohort from the United States. J Stroke Cerebrovasc Dis 17(2):49-54

3. Iurlaro S, Beghi E, Massetto N, Guccione A, Autunno M, Colombo B, Di Monda T, Gionco M, Cortelli P, Perini F, D'Onofrio F, Agostoni E (2004) Does headache represent a clinical marker in early diagnosis of cerebral venous thrombosis? A prospective multicentre study. Neurol Sci 25(Suppl 3):S298S299

4. Cumurciuc R, Crassard I, Sarov M, Valade D, Bousser MG (2005) Headache as the only neurological sign of cerebral venous thrombosis: a series of 17 cases. J Neurol Neurosurg Psychiatry 76(8):1084-1087

5. de Bruijn SF, Stam J, Kappelle LJ (1996) Thunderclap headache as first symptom of cerebral venous sinus thrombosis. CVST Study Group. Lancet 348(9042):1623-1625

6. Slooter AJ, Ramos LM, Kappelle LJ (2002) Migraine-like headache as the presenting symptom of cerebral venous sinus thrombosis. J Neurol 249(6):775-776

7. Todorov L, Laurito CE, Schwartz DE (2005) Postural headache in the presence of cerebral venous sinus thrombosis. Anesth Analg 101(5):1499-1500

8. Rodríguez S, Calleja S, Morís G (2008) Cluster-like headache heralding cerebral venous thrombosis. Cephalalgia 28(8):906907

9. Agostoni E (2004) Headache in cerebral venous thrombosis. Neurol Sci 25(Suppl 3):S206-S210

10. Wasay M, Azeemuddin M (2005) Neuroimaging of cerebral venous thrombosis. J Neuroimaging 15:118-128

11. Ravishankar K (2006) Incidence and pattern of headache in cerebral venous thrombosis. J Pak Med Assoc 56(11):561-564 